\title{
Increasing the brightness of red fluorescent proteins by saturation mutagenesis
}

\author{
Erin Nguyen ${ }^{1}$, Antonia Pandelieva ${ }^{2}$ and Roberto A. Chica ${ }^{1,3 *}$
}

\begin{abstract}
Red fluorescent proteins (RFPs) are genetically-encoded fluorophores that are widely used for in vivo imaging. For all applications of RFPs, brighter variants are desired. Previously, we improved the brightness of mRojoA, a red-shifted mutant of the widely-used RFP $m$ Cherry, by designing a triple-decker motif of aromatic rings around its chromophore. This yielded the brighter variant mRojo-VHSV, which contains a triple-decker motif consisting of His and Tyr side chains that pack against the chromophore. This improved chromophore packing resulted in an approximately 3-fold brightness increase at physiological $\mathrm{pH}$. However, the His side chain in the triple-decker motif of mRojo-VHSV adopted a perpendicular arrangement to the other two, which may result in a suboptimal packing arrangement. To further improve chromophore packing in mRojo-VHSV, we performed saturation mutagenesis of residues surrounding its triple-decker motif (positions 62, 97, 165, and 199). Using a microplate fluorescence screening assay, a total of 376 colonies were screened for improved brightness. The brightest mutant found, L199M, was expressed and purified, and its spectral properties were characterized in detail. We found that the quantum yield of this variant was improved by two-fold, resulting in a two-fold brightness increase compared to mRojo-VHSV as well as a 5.3-fold increase in brightness compared to mRojoA. The L199M improved variant is the basis for continued engineering with the goal of further improving the spectral properties of this family of RFPs.
\end{abstract}

Keywords: Protein engineering; Quantum yield; Bathochromic shift; Library screening; Rational design

\section{Résumé}

Les protéines rouges fluorescentes (PRF) sont des fluorophores génétiquement codés qui sont largement utilisés pour l'imagerie in vivo. On désire obtenir des variantes plus lumineuses pour toutes les applications des PFR. Auparavant, nous avons amélioré la brillance de $m$ RojoA, un mutant décalé vers le rouge de la PFR populaire mCherry, par la conception d'un motif à trois étages d'anneaux aromatiques autour du chromophore. Le résultat fut une variante plus brillante, mRojo-VHSV, qui contient un motif à trois étages composé de chaînes latérales His et Tyr qui se plaquent contre le chromophore. Cet empaquetage du chromophore amélioré a abouti à une augmentation de luminosité d'environ 3 fois, au pH physiologique. Cependant, la chaîne de His dans le motif à trois étages de mRojo-VHSV a adopté un arrangement perpendiculaire aux deux autres, ce qui peut entraîner un arrangement d'empaquetage sous-optimal. Pour améliorer davantage l'empaquetage du chromophore de mRojo-VHSV, nous avons effectué la mutagenèse de saturation des résidus entourant son motif à trois étages (positions 62, 97, 165 et 199). Afin de cribler pour une luminosité améliorée, nous avons utilisé un essai de fluorescence avec microplaques. Au total, 376 colonies ont été criblées pour une luminosité améliorée. Le mutant le plus brillant, soit L199M, a été exprimé, purifié et caractérisé afin de déterminer ses propriétés spectrales détaillées. Nous avons constaté que le rendement quantique de cette variante est deux fois plus supérieur qu'initialement, résultant en une augmentation de la luminosité de deux fois par rapport à mRojo-VHSV, ainsi que d'une augmentation de la luminosité de 5,3 fois par rapport à mRojoA. La variante L199M améliorée sert comme base pour la poursuite de la recherche qui vise à améliorer davantage les propriétés spectrales de cette famille de PRF.

Mots Clés: Ingénierie des protéines; Rendement quantique; Déplacement bathochrome; Criblage de librairie; Conception rationnelle 


\section{Introduction}

Red fluorescent proteins (RFPs) are members of the green fluorescent protein (GFP) superfamily of fluorescent proteins that emit light in the red and far-red regions of the visible spectrum (580-680 nm) (2). They differ from other members of this superfamily by the presence of an acylimine group conjugated to the standard p-hydroxybenzilideneimidazolinone chromophore of GFP, which extends the size of the chromophore $\pi$ system, leading to longer emission wavelengths. The longer emission wavelengths of RFPs make them particularly useful for whole-body imaging of research model animals since cells are more transparent to red light (3), allowing deep tissue imaging. An additional benefit of the longer emission wavelength of RFPs is its lower energy, which reduces phototoxicity and thereby enables longer experiments (2). However, RFPs tend to be less bright than other members of the GFP superfamily (4), making it desirable to develop brighter variants through protein engineering.

Previously, we improved the brightness of mRojoA, a red-shifted and dim variant of the widely used monomeric RFP mCherry (5), by engineering a triple-decker motif of aromatic rings surrounding its chromophore. This resulted in a variant displaying up to 3 -fold increases in brightness: mRojo-VHSV with mutations at position 16 (V), $63(\mathrm{H}), 143(\mathrm{~S})$ and $163(\mathrm{~V})$. Notably, the conformation of the side chain of residue His63 located directly above the chromophore resulted in a perpendicular arrangement between its aromatic ring and the phenolate group of the chromophore at a distance consistent with a weak edge-to-face -stacking interaction. (1)

Here, our goal was to optimize the triple-decker motif to obtain a parallel arrangement of aromatic rings and maximize packing. We hypothesized that such an arrangement would lead to an increase in the RFP brightness and a bathochromic shift in emission wavelength since it would improve the $\pi$-stacking interactions of the chromophore with nearby aromatic residues to further increase its conjugation. (6) Optimization of the motif was accomplished by performing saturation mutagenesis of residues S62, M97, L165 and L199, which surround the triple-decker motif in mRojo-VHSV (Figure 1). The brightest mutant found, L199M, displayed a two-fold brightness increase compared to mRojo-VHSV as well as a 5.3-fold increase in brightness compared to mRojoA, which was mostly caused by an improved quantum yield.

\section{Results}

Screening of the saturation libraries (Table 1) revealed that positions 62 and 199 are less tolerant to mutations

\footnotetext{
${ }^{*}$ Correspondence: rchica@uottawa.ca

${ }^{1}$ Department of Chemistry and Biomolecular Sciences, University of Ottawa, 10 Marie Curie, K1N 6N5, Ottawa, Canada

Full list of author information is available at the end of the article
}

Table 1: Whole cell screening assay results.

\begin{tabular}{lcccc}
\hline \multicolumn{4}{c}{ Library $^{a}$} \\
\hline & S62X & M97X & L165X & L199X \\
Fluorescent $^{b}$ & 12 & 71 & 70 & 27 \\
Bright $^{c}$ & 0 & 0 & 1 & 2 \\
${ }^{a}$ For each library, 94 variants were screened. \\
${ }^{b}$ Variants displaying a fluorescence intensity greater than \\
125\% that of the negative control. \\
${ }^{c}$ Variants displaying a fluorescence intensity greater than \\
$125 \%$ that of the parent protein, mRojo-VHSV.
\end{tabular}

with less than $30 \%$ of the variants displaying fluorescence above that of negative control cells containing an empty pET-11a vector. In contrast, approximately $75 \%$ of the variants from saturation libraries 97 and 165 were fluorescent, suggesting that these positions are more tolerant to mutation. However, none of the fluorescent mutants displayed a significant red shift in their emission wavelength.

Three brighter variants (i.e. fluorescence intensity greater than $125 \%$ that of the mRojo-VHSV, the parent protein) were identified from the screening. The brighter mutant from library L165X was found to be L165F, while both brighter variants from the L199X library contained the same mutation (L199M). We characterized the spectral properties of mRojo-VHSV L165F and L199M and compared them with those of the mRojo-VHSV parent RFP (Table 2 and Figure 2).

The mutation L165F conserved the excitation wavelength from its parent protein, but a slight red-shift of 5 $\mathrm{nm}$ was observed for the emission wavelength (Figure 2, panel I). This discrepancy with the screening results is likely caused by the lower signal to noise ratio of these whole cell assays versus the in vitro assays using purified protein. The extinction coefficient of the L165F mutant decreased by a factor of 1.8 while the quantum yield remained unchanged, resulting in an overall brightness decrease of 1.8-fold. False positives such as this mutation are possible since the screening was done to target variants with increased brightness in whole cells. Such an increase can also be caused by a higher concentration of RFPs in the cells or a more efficient chromophore maturation. By analyzing our computational model, we hypothesize that the presence of the new phenylalanine side chain at position 165 cannot allow the H63 side chain to be in the same conformation because of steric hindrance. Interestingly, the Phe side chain introduced by the L165F mutation is located directly above the His63 side chain, making it possible to form a quadruple-decker motif with the current triple-decker motif (Figure 3). Such a motif has not yet been observed in fluorescent proteins.

The L199M mutation resulted in a $4 \mathrm{~nm}$ blue shift of the excitation and emission wavelengths (Figure 2, 


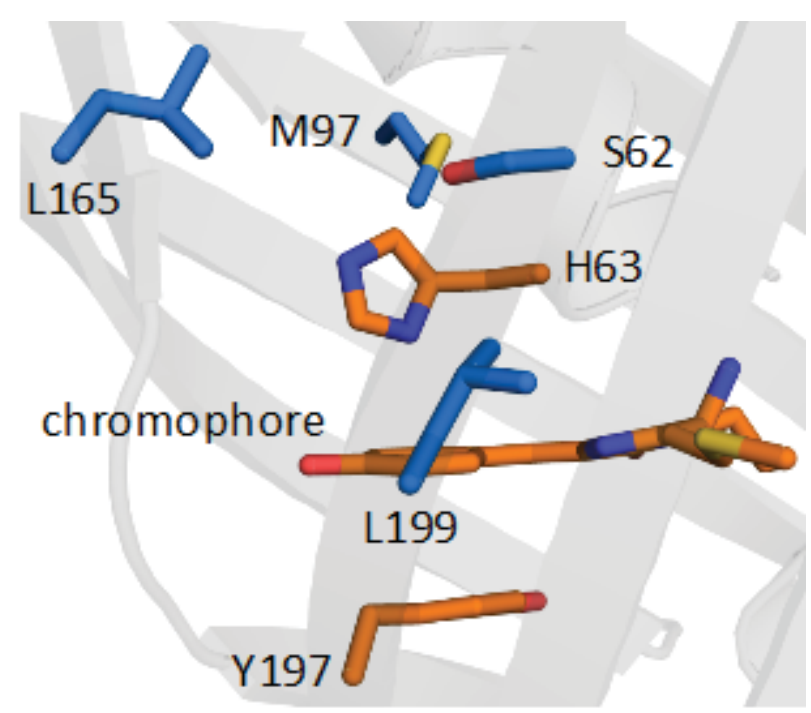

Figure 1: Model of $\mathbf{m R o j o - V H S V}$ triple decker motif. The motif is formed by, in orange, H63 and Y197 with the chromophore in the middle, and the positions selected for saturation mutagenesis are in blue (S62, M97, L165, and L199). Model is based on PDB ID: 5H87 (1)

Table 2: $\quad$ Spectral properties of various RFPs.

\begin{tabular}{lccccc}
\hline RFP & $\lambda_{e x}(\mathrm{~nm})$ & $\lambda_{e m}(\mathrm{~nm})$ & $\epsilon\left(\mathrm{mM}^{-1} \mathrm{~cm}^{-1}\right)$ & $\varphi \times 10^{-2}$ & Brightness $(\%$ mRojo-VHSV $)$ \\
\hline mRojo-VHSV & $592 \pm 1$ & $622 \pm 2$ & $117 \pm 13$ & $4.0 \pm 0.4$ & 100 \\
mRojo-VHSV L165F & $593 \pm 2$ & $627 \pm 1$ & $66 \pm 8$ & $4.1 \pm 0.2$ & 58 \\
mRojo-VHSV L199M & 588 & 618 & 118 & $9 \pm 1$ & 226 \\
mRojo-VHSV FM & $588 \pm 1$ & $619 \pm 1$ & 66 & $7.5 \pm 0.3$ & 105
\end{tabular}

Experiments were performed in triplicate (except for mRojo-VHSV L199M) from three independent proteins preparations at a $\mathrm{pH}$ of 7.4 .
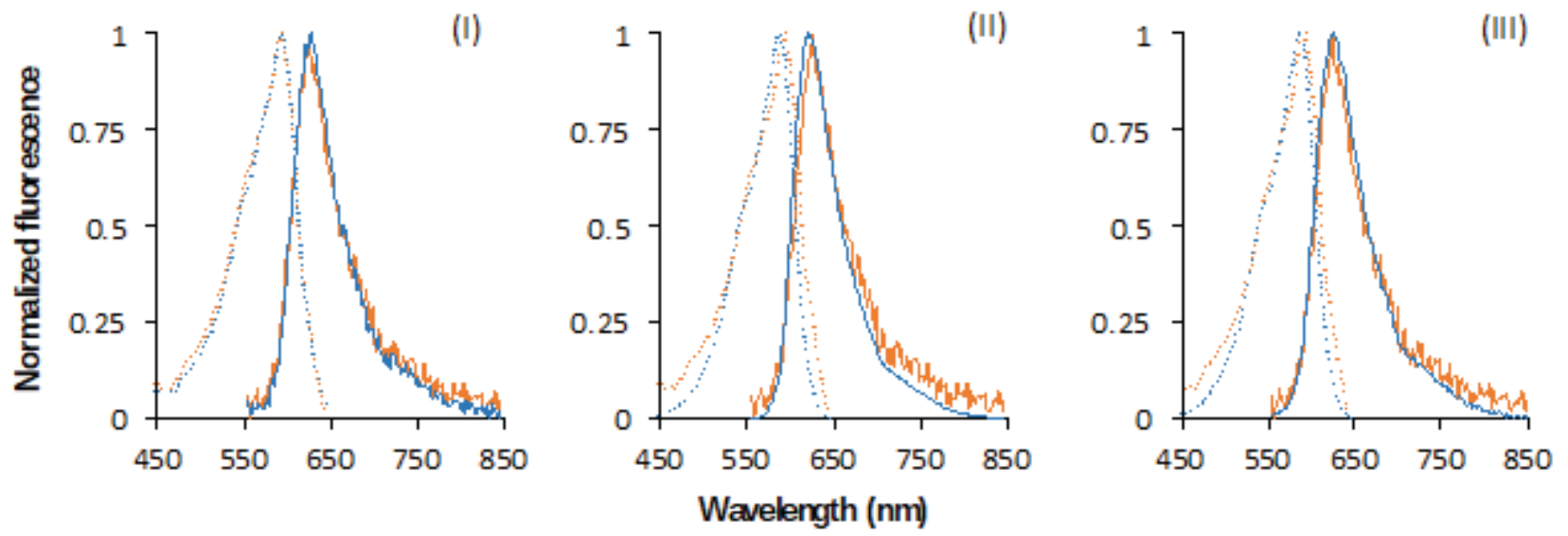

Figure 2: $\quad$ Fluorescence spectra. (I) mRojo-VHSV L165F, (II) mRojo-VHSV L199M, (III) mRojo-VHSV FM. mRojo-VHSV spectra are in orange while those of mutants are in blue. Excitation and emission spectra are represented as dashed and solid lines, respectively.

panel II). Contrary to the L165F mutation, the L199M mutation did not affect the extinction coefficient but increased the quantum yield by 2.3 -fold. Because of this, an overall increase in brightness of the same magnitude was observed.

Since the L165F mutation causes an emission wavelength red shift while having the potential to create a 


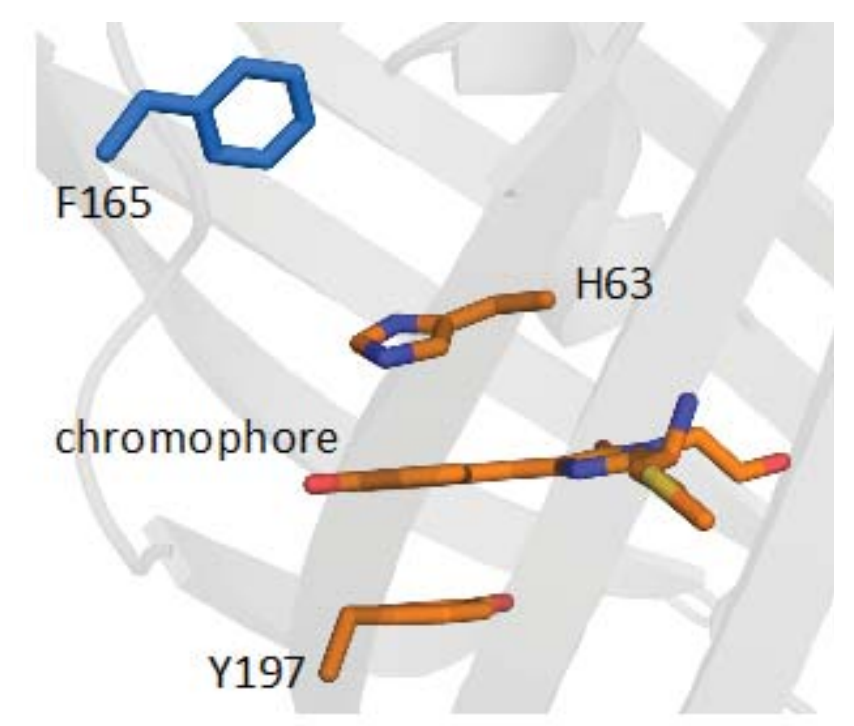

Figure 3: Computational model of the structure of mRojo-VHSV L165F. The triple-decker motif is represented in orange ( $\mathrm{H} 63$ and $\mathrm{Y} 197$ with the chromophore in the middle) and the F165 residue in blue. Note the possible quadruple-decker motif. PDB ID: $5 \mathrm{H} 87$ (1).

quadruple-decker motif of aromatic rings, we combined it with the brightness increasing L199M mutation to create the mRojo-VHSV FM double mutant. As was observed with the L199M single mutant, excitation and emission wavelengths were blue-shifted by $4 \mathrm{~nm}$. (Figure 2, panel III) In addition, the extinction coefficient of the double mutant decreased by a factor of 1.8 , as in the $\mathrm{L} 165 \mathrm{~F}$ single mutant, while the quantum yield increased by a factor of 1.9. Overall, a small (5\%) increase in brightness was observed. Even though the quantum yield of mRojoVHSV FM was lower than that of the single mutant L199M, this variant can potentially form a quadrupledecker motif of aromatic rings while restoring the loss of brightness caused by the L165F mutation.

\section{Discussion}

Even though the screening results indicated that the single mutant L165F is brighter than mRojo-VHSV, spectroscopic characterization of the purified protein revealed that this was not the case. However, a small red shift of the emission wavelength was observed. This could be due to parallel $\pi$-stacking from residue $\mathrm{H} 63$ with the chromophore, as such an interaction was predicted by quantum mechanics to cause a similar emission red shift in GFP (6). However, it is unclear how this mutation decreased the extinction coefficient. A crystal structure of mRojo-VHSV L165F is required to verify the orientation of the histidine side chain relative to the chromophore.

The increase in brightness observed for the L199M mutant could result from a similar process as was seen in the

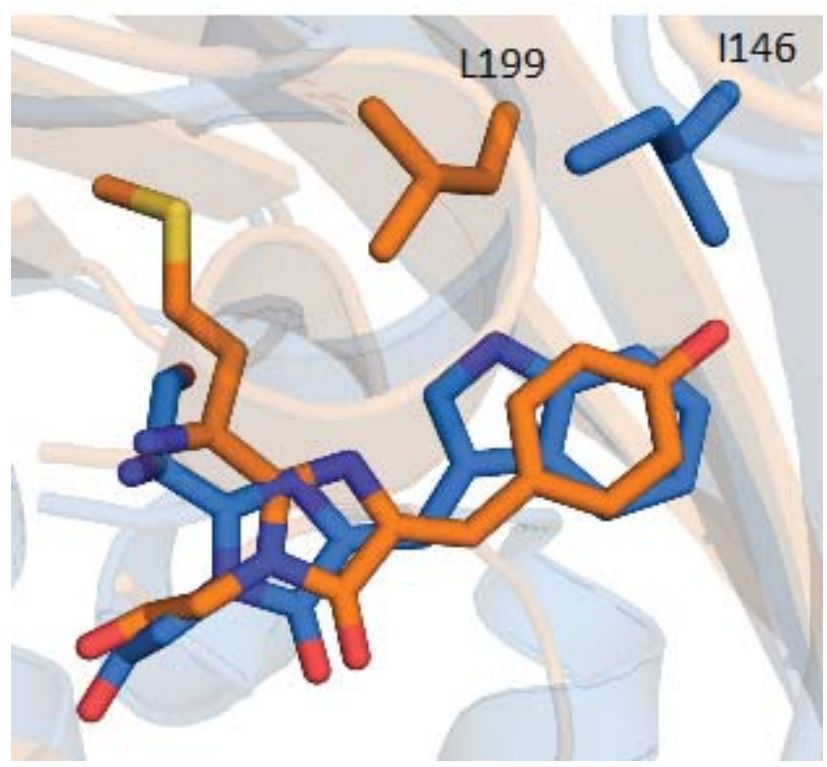

Figure 4: Overlay of the positioning of L199 in mRojo-VHSV and of $\mathbf{1 1 4 6}$ in mTurquoise.

mRojo-VHSV is shown in orange while mTurquoise is shown in blue. PDB ID: 5H87, mRojo-VHSV (1) and 4AR7, mTurquoise (7)

mTurquoise 2 cyan fluorescent protein, which displays a quantum yield increase of $10 \%$ but no change in extinction coefficient relative to its parent mTurquoise. Goedhart et al. observed that the single mutation I146F increased the number of van der Waals interactions with the chromophore and surrounding residues when it was introduced into mTurquoise. This improved packing led to the rigidification of a flexible strand in mTurquoise and of the chromophore, resulting in a quantum yield increase (7). A similar process could have happened in mRojo-VSHV L199M since L199 in mRojo-VHSV and I46 in mTurquoise occupy similar positions relative to the chromophore (Figure 4).

When combining the two mutations, we did not observe a quantum yield increase as was seen in the L199M point mutant, while the extinction coefficient was within error to that of the L165F single mutant, leading us to conclude that the mutations are not additive. The decrease in quantum yield when adding the L165F mutation to the L199M mutation could indicate that it contributes to the destabilization of the chromophore, making it more flexible. Combinatorial mutagenesis at these sites may help to identify synergistic mutations that would increase the quantum yield and overall brightness.

In conclusion, saturation mutagenesis of amino acids surrounding the triple-decker motif of mRojo-VHSV led to an increase in brightness of up to 2.3-fold from the single mutation, L199M. It was also found that the mutation L165F has the potential to form a quadruple-decker 
motif which would be novel in this protein family. However, this single mutation decreased the brightness of the RFP. Combining both mutations into mRojo-VHSV FM created an RFP with the putative quadruple-decker motif while maintaining the brightness observed in mRojoVHSV. This combined mutant could be used as a starting point in directed evolution to further increase the brightness of an RFP with a potential quadruple-decker motif.

\section{Materials and Methods Mutagenesis and cloning}

Saturation mutagenesis was performed on the mRojoVHSV gene by overlap extension using Vent DNA polymerase (New England BioLabs). External primers were used in combination with complementary sets of primers containing the NNS degenerate codon in individual polymerase chain reactions (PCR). The resulting overlapping fragments were agarose gel-purified (Omega Bio-tek) and recombined by overlap extension PCR. The amplicons were digested with NdeI and BamHI (New England BioLabs) and gel-purified to be ligated into a digested and dephosphorylated - via treatment with Antarctic phosphatase (New England BioLabs) - pET-11a vector (Novagen) using T4 DNA ligase (New England BioLabs). Constructs were transformed into chemically competent E. coli BL21-Gold(DE3) cells (Agilent). Plasmids were extracted from transformed cells using E.Z.N.A Plasmid Mini Kit I (Omega Bio-tek) and were verified by sequencing the entire reading frame.

\section{Screening}

Cells were grown in Overnight Express Instant Terrific Broth (TB) at $37^{\circ} \mathrm{C}$ with shaking in 96-well plates (Nunc). Following overnight expression, cells were harvested by centrifugation. Pellets were washed using phosphate-buffered saline (PBS) at $\mathrm{pH} 7.4$ then left at $4^{\circ} \mathrm{C}$ for three days to allow for chromophore maturation. Screening of the saturation mutagenesis libraries was performed using a Tecan Infinite M1000 plate reader. The excitation spectra (with emission at $620 \mathrm{~nm}$ ) and the emission spectra (with excitation at $535 \mathrm{~nm}$ ) were obtained from whole cells resuspended in PBS at pH 7.4.

\section{Protein expression and purification}

E. coli BL21-Gold(DE3) cells containing the His-tagged RFP genes were inoculated and grown overnight $\left(37^{\circ} \mathrm{C}\right.$, $200 \mathrm{rpm}$ ) in a Luria-Bertani (LB) broth supplemented with $100 \mu \mathrm{M}$ of ampicillin. $10 \mathrm{~mL}$ of the overnight culture was transferred into $500 \mathrm{~mL}$ of LB broth supplemented with $100 \mu \mathrm{M}$ of ampicillin. Cells were grown at $37^{\circ} \mathrm{C}, 200 \mathrm{rpm}$ until the cultures reached an optical density at $600 \mathrm{~nm}$ between 0.5 and 0.7 . Protein expression was induced using isopropyl -D-1-thiogalactopyranoside
(IPTG) at a final concentration of $1 \mathrm{mM}$. Cells were induced overnight at $16^{\circ} \mathrm{C}$ with shaking at $200 \mathrm{rpm}$, then they were harvested by centrifugation. Pellets were resuspended in lysis buffer (100 mM phosphate buffer $\mathrm{pH} 8.0$ supplemented with $5 \mathrm{mM}$ imidazole) and lysed with an EmulsiFlex-B15 cell disruptor (Avestin). Proteins were purified by immobilized metal affinity chromatography using Profinity IMAC Ni-charged resin (Bio-rad) following the manufacturer's protocol. Eluted fractions containing the protein of interest were desalted by gel filtration using EconoPAC 10 DG columns (Bio-rad) into a buffer of $250 \mathrm{mM}$ HEPES with $100 \mathrm{mM} \mathrm{NaCl}$ (pH 7.25).

\section{Spectroscopic Characterization}

Absorption, emission and excitation spectra were recorded in PBS at pH 7.4 using a Tecan Infinite M1000 plate reader. Quantum yields $(\varphi)$ were determined from the integrated fluorescence intensity from excitation at 535 nm compared to samples of mCherry $(\varphi=0.22,(8))$ for the range of emission from 550 to $800 \mathrm{~nm}(9)$. The molar extinction coefficients were calculated by the dynamic difference method described by Kredel et al. to take into account the presence of green chromophore. (10)

\section{Competing interests}

The authors declare that they have no competing interests.

\section{Acknowledgements}

RAC acknowledges an Early Research Award from the Ontario Ministry of Economic Development \& Innovation, and grants from the Natural Sciences and ENgineering Research Council of Canada (NSERC), the Ontario Research Fund, and the Canada Foundation for Innovation. EN was the recipient of an Undergraduate Student Research Award from NSERC.

\section{Author details}

${ }^{1}$ Department of Chemistry and Biomolecular Sciences, University of Ottawa, 10 Marie Curie, K1N 6N5, Ottawa, Canada. ${ }^{2}$ Cytokines Division, Health Canada, 70 Colombine Driveway, K1A 0K9, Ottawa, Canada. ${ }^{3}$ Centre for Catalysis Research and Innovation, University of Ottawa, 30 Marie Curie, K1N 6N5, Ottawa, Canada.

References

1. A. T. Pandelieva, et al., ACS Chem. Biol. 11, 508 (2016).

2. D. M. Chudakov, M. V. Matz, S. Lukyanov, K. A. Lukyanov, Physiol. Rev. 90, 1103 (2010).

3. G.-J. Kremers, S. G. Gilbert, P. J. Cranfill, M. W. Davidson, D. W. Piston, Journal of cell science 124, 157 (2011).

4. K. Nienhaus, G. U. Nienhaus, Chem Soc Rev 43, 1088 (2014).

5. R. A. Chica, M. M. Moore, B. D. Allen, S. L. Mayo, Proc. Natl. Acad. Sci. U.S.A. 107, 20257 (2010).

6. B. L. Grigorenko, A. V. Nemukhin, I. V. Polyakov, A. I. Krylov, J Phys Chem Lett 4, 1743 (2013)

7. J. Goedhart, et al., Nat Commun 3, 751 (2012).

8. N. C. Shaner, et al., Nat. Biotechnol. 22, 1567 (2004)

9. A. Williams, S. Winfield, J. Miller, Analyst 108, 1067 (1983).

10. S. Kredel, et al., Chem. Biol. 15, 224 (2008). 